\title{
Análise conceitual: considerações metodológicas
}

\author{
Conceptual analysis: methodological considerations
}

Análisis conceptual: consideraciones metodológicas

\author{
Maria das Graças Melo Fernandes', Maria Miriam Lima da Nóbrega", \\ Telma Ribeiro Garcia"', Kátia Nêyla de Freitas Macêdo-Costa' \\ ' Universidade Federal da Paraíba, Departamento de Enfermagem Clínica, \\ Programa de Pós-Graduação em Enfermagem. João Pessoa-PB. Brasil. \\ "Universidade Federal da Paraíba, Departamento de Enfermagem de Saúde Pública e Psiquiatria, \\ Programa de Pós-Graduação em Enfermagem. Pesquisadora do CNPq. João Pessoa-PB. Brasil. \\ I" Universidade Federal da Paraíba, Departamento de Enfermagem de Saúde Pública e Psiquiatria (Aposentada). \\ João Pessoa-PB. Brasil.
}

Submissão: 04-05-2010 Aprovação: 10-12-2011

\section{RESUMO}

Conceitos referem-se a fenômenos que ocorrem na natureza ou no pensamento, sendo essenciais no desenvolvimento de pesquisas, assim como na construção de teorias. Para que cumpram esse papel na construção do conhecimento científico, é oportuno que sejam analisados periodicamente, visando, principalmente, seu contínuo aprimoramento. Apesar da existência de diferentes modelos de análise conceitual, esta metodologia, no geral, não tem sido bem compreendida pela Enfermagem, especialmente em nossa realidade. Assim sendo, neste artigo de reflexão, objetivamos descrever algumas considerações metodológicas e uma abordagem prática sobre os métodos propostos por Walker e Avant, Rodgers e Schwartz-Barcott e Kim, com vistas a proporcionar aos enfermeiros maior compreensão desse processo.

Descritores: Conceito; Metodologia; Enfermagem.

\begin{abstract}
Concepts are terms referring to phenomena that occur in nature or in thought, which are essential in developing research, as well as in the construction of theories. To fulfill this role in the construction of scientific knowledge, it is appropriate for them to be reviewed periodically, aiming mainly at its improvement. Despite the existence of different models of conceptual analysis, this methodology, in general, has not been well understood by nursing, especially in our reality. Therefore, in this article, of reflection, we describe some methodological considerations, including a practical approach on the methods proposed by Walker and Avant, Rodgers and Schwartz-Barcott and Kim, in order to give nurses greater understanding of this process.
\end{abstract}

Key words: Concept; Methodology; Nursing.

\section{RESUMEN}

Los conceptos son términos referentes a los fenómenos que ocurren en la naturaleza o en el pensamiento, siendo esenciales en el desarrollo de investigaciones, así como en la construcción de teorías. Para que ellos cumplan su papel en la construcción del conocimiento científico, es oportuno que sean analizados periódicamente, teniendo como objetivo, principalmente, su continua mejoría. A pesar de la existencia de diferentes modelos de análisis conceptual, esta metodología, en general, no ha sido bien comprendida por la Enfermería, especialmente en nuestra realidad. De esta manera, en este artículo de reflexión, objetivamos describir algunas consideraciones metodológicas, incluyendo un abordaje práctico, acerca de los métodos propuestos por Walker y Avant, Rodgers y Schwartz-Barcott y Kim, con el fin de proporcionar a los enfermeros mayor comprensión acerca de ese proceso.

Palabras clave: Concepto; Metodología; Enfermería. 


\section{INTRODUÇÃO}

Um conceito é uma idéia ou construção mental elaborada acerca de um fenômeno(1). São termos referentes aos fenômenos que ocorrem na natureza ou no pensamento ${ }^{(2)}$. São representações cognitivas, abstratas, de uma realidade perceptível formada por experiências diretas ou indiretas ${ }^{(3)}$. Os conceitos podem ser empíricos ou concretos (observados pelos sentidos) ou abstratos (não observáveis). Sua função primária é permitir que indivíduos possam descrever situações e se comunicar efetivamente.

Como representações de uma dada realidade, os conceitos possuem atributos de caráter dinâmico, mutáveis na dimensão temporal e contextual, sendo sua evolução influenciada pelo uso e aplicação. Desse modo, para que cumpram seu papel na construção do conhecimento científico, é oportuno que seus atributos essenciais e, consequentemente, suas definições sejam analisados periodicamente, visando, principalmente, seu contínuo aprimoramento. Os conceitos "não são esculpidos em pedras, sua análise e entendimento mudam com o tempo, não devendo ser considerados como um produto finalizado"(4:38).

A revisão dos atributos essenciais e da definição dos conceitos ocorre por meio de análise conceitual, processo que incorpora um exame completo dos elementos básicos que compõem um pensamento, idéia ou noção ${ }^{(5)}$. Essa análise deve ser empreendida quando um conceito, já introduzido, definido e clarificado na literatura de uma disciplina específica, necessita de estudo adicional para movê-lo ao próximo nível de desenvolvimento, de modo a torná-lo aplicável eficazmente na pesquisa e na prática dessa disciplina, ampliando seu entendimento entre aqueles que o utilizam ${ }^{(2)}$.

No tocante ao método, temos na literatura pertinente vários modelos para análise conceitual, os quais apresentam como meta principal o exame dos atributos críticos de um conceito particular. Apesar da existência de diferentes modelos de análise conceitual, esta metodologia, no geral, não tem sido bem compreendida pela Enfermagem, especialmente em nossa realidade. Assim sendo, neste estudo, objetivamos descrever algumas considerações metodológicas e um exemplo de aplicação prática dos métodos propostos por Walker e Avant ${ }^{(4)}$ por Rodgers( ${ }^{(6)}$ e por Schwartz-Barcott e Kim ${ }^{(7)}$ para análise conceitual, com vistas a proporcionar aos enfermeiros maior compreensão acerca desse processo.

\section{MÉTODO DE ANÁLISE CONCEITUAL DE WALKER E AVANT}

Este método para análise de conceito, fundamentado em Wilson $^{(8)}$, é o mais usado na Enfermagem. Inclui oito passos, que serão descritos sequencialmente, apesar de alguns ocorrerem simultaneamente, para facilitar a compreensão dos leitores ${ }^{(4)}$.

Seleção do conceito - deve refletir o tópico ou a área de maior interesse, sendo recomendável a escolha de um conceito que esteja ligado à área de experiência profissional (prática, pesquisa, ensino, administração) e que tenha despertado atenção e preocupação na pessoa.
Determinação dos objetivos da análise conceitual - refere-se à finalidade da análise conceitual que se pretende realizar: desenvolvimento de instrumentos de pesquisa ou de uma definição operacional (real); desenvolvimento de diagnóstico de enfermagem; esclarecimento de conceitos vagos ou imprecisos utilizados na prática profissional; refinamento de um conceito quando ele aparenta ter múltiplos significados; ajuste entre a definição do conceito e sua aplicação prática.

Identificação dos possíveis usos do conceito - trata-se de uma busca na literatura para se ter uma ideia de como o conceito em questão está sendo enfocado ou aplicado. Durante esse passo, deve-se considerar os usos implícitos e explícitos do conceito, sendo aconselhável que se extrapole a literatura médica e de enfermagem (ver dicionários, enciclopédias, livros, artigos científicos, instrumentos ou escalas de medidas de elementos presentes no conceito). Isso evita vieses na compreensão da sua natureza, ou limitação na utilidade dos resultados da análise. Após ter identificado os usos do conceito, tanto no campo científico quanto no senso comum, é importante decidir quais aspectos serão considerados, se todos ou apenas aqueles pertinentes ao campo científico.

Determinação dos atributos críticos ou essenciais - representa um passo fundamental na análise do conceito, em que se identificam palavras ou expressões que aparecem repetidamente na literatura, que mostram a essência do conceito. Esses atributos constituem características que expressam o conceito, as quais atuam como elementos para diagnósticos diferenciais, isto é, para discriminar o que é uma expressão do conceito daquilo que não é. Nos casos em que o conceito é muito abstrato, os seus atributos, geralmente, têm também alto grau de abstração. Vale destacar que, em qualquer modelo de análise conceitual, a identificação dos elementos do conceito na literatura é feita utilizando-se a técnica de análise de conteúdo. Para isso, essas questões podem facilitar a investigação: como o autor define o conceito? quais características ou atributos do conceito são apontados por ele? que ideias o autor discute sobre o conceito?

Construção de um caso modelo - elaboração de um exemplo, baseado na vida real, do uso do conceito, que inclua seus atributos essenciais. O caso deve ser o mais paradigmático possível.

Desenvolvimento de outros casos: limítrofes, relacionados, contrários inventados e ilegítimos. Servem para auxiliar na decisão quanto aos atributos essenciais do conceito. Entre esses casos, chamamos atenção para dois deles que, no nosso entendimento, melhor cumprem a meta ora exposta: o caso contrário e o caso limítrofe. O caso contrário provê exemplo do "não conceito". Já o caso limítrofe, constitui aquele evento ou instância que contém alguns dos atributos essenciais do conceito sob análise, mas não todos eles.

Identificação de antecedentes e consequências do conceito - é o levantamento de incidentes ou eventos que acontecem a priori ao fenômeno (necessários para a sua ocorrência) e a posteriori (eventos ou situações que surgem ou resultam da presença do fenômeno). Lembrar a observação do critério de exclusividade, algo não pode ser, concomitantemente, um atributo, um antecedente ou consequência. 
Definição de referências empíricas para os atributos essenciais - referentes empíricos são categorias ou classes de fenômenos observáveis que, quando presentes, demonstram a ocorrência do conceito, possibilitando, assim, sua definição operacional. Em muitos casos, os atributos são idênticos às referências empíricas. Quando os conceitos são abstratos (autoestima, tristeza), seus indicadores empíricos não são diretamente observáveis, dependem de medidas indiretas.

No tópico que se segue apresentaremos exemplo de uma análise do conceito fadiga, em que se utilizou o modelo ora descrito $^{(9)}$.

Seleção do conceito e objetivo da análise: as autoras selecionaram para análise o conceito fadiga, em virtude de observar uma incongruência relativa à concepção e à operacionalização do termo por parte dos profissionais de saúde que lidam com o fenômeno tanto na prática quanto na pesquisa. Considerando isso, foi delimitado como objetivo da análise clarificar o conceito a fim de estabelecer fundamentos para a sua aplicação prática.

a) Identificação dos usos do conceito fadiga no âmbito da literatura.

\begin{tabular}{|c|c|}
\hline Psicologia & $\begin{array}{l}\text { Estado de desgaste relacionado à redução da } \\
\text { motivação. }\end{array}$ \\
\hline Enfermagem & $\begin{array}{l}\text { Uma sensação opressiva e sustentada de exaustão } \\
\text { e de capacidade diminuída para realizar trabalho } \\
\text { físico e mental no nível habitual. } \\
\text { Sintoma subjetivo, desagradável que incorpora } \\
\text { toda sensação do corpo variando de cansaço a } \\
\text { exaustão, criando uma condição geral de falta de } \\
\text { alívio, que interfere na capacidade do indivíduo } \\
\text { realizar suas habilidades normais. } \\
\text { Experiência aguda ou crônica caracterizada pelo } \\
\text { desempenho não efetivo de tarefas, inadequação } \\
\text { auto-percebida, aversão à atividades, cansaço ou } \\
\text { sensação de fraqueza, desconforto. }\end{array}$ \\
\hline $\begin{array}{l}\text { Educação } \\
\text { Física }\end{array}$ & $\begin{array}{l}\text { Declínio na capacidade de gerar tensão } \\
\text { muscular com a estimulação repetida. }\end{array}$ \\
\hline Index Medicus & $\begin{array}{l}\text { Estado de desgaste que segue um período de } \\
\text { esforço, mental ou físico caracterizado por } \\
\text { uma diminuição da capacidade de trabalhar } \\
\text { e redução da eficiência para responder a um } \\
\text { estímulo. }\end{array}$ \\
\hline Odontologia & $\begin{array}{l}\text { A fadiga refere-se ao 'desgaste' de materiais de } \\
\text { próteses parciais, geralmente gerado por falha } \\
\text { no próprio material ou por efeito de cargas } \\
\text { repetidas. }\end{array}$ \\
\hline Engenharia & $\begin{array}{l}\text { Fadiga é um modo prevalente de falência } \\
\text { de componentes estruturais ocasionado por } \\
\text { períodos de estresse. }\end{array}$ \\
\hline $\begin{array}{l}\text { Medical } \\
\text { Dictionary }\end{array}$ & $\begin{array}{l}\text { Estado com aumento do desconforto e } \\
\text { diminuição da eficiência resultante de um } \\
\text { esforço prolongado ou excessivo. }\end{array}$ \\
\hline
\end{tabular}

Figura 1 - Definições do conceito fadiga presentes na literatura pesquisada. b) Identificação dos atributos essenciais: a análise da literatura permitiu identificar que a fadiga é evidenciada por cansaço, desgaste, fraqueza, astenia, diminuição da capacidade funcional ou da capacidade de realizar atividades diárias, falta de recursos/energia/capacidade latente, redução da eficiência para responder a um estímulo, desconforto, sonolência, diminuição da motivação, aversão a atividades, sofrimento e necessidade extrema de descanso. A partir da identificação dos atributos da fadiga, foi desenvolvida a seguinte definição para o fenômeno: "estado de desgaste que segue um período de esforço mental ou físico, caracterizado por diminuição da capacidade de trabalhar e redução da eficiência para responder a um estímulo" (9).

c) Construção de um caso-modelo, que apresenta todos os atributos essenciais do conceito, anteriormente mencionados: "executivo, com diagnóstico recente de câncer de cabeça e pescoço, após x sessões de radioterapia, começa a apresentar necessidade de deixar o trabalho mais cedo que o habitual e não consegue mais desenvolver todas as atividades domésticas pelas quais era responsável, por sentir-se muito cansado. Mantém-se em repouso, buscando descanso, durante grande parte do dia e refere que mesmo assim não consegue recuperar a disposição e energia necessárias para o funcionamento habitual prévio" ${ }^{(9)}$.

d) Desenvolvimento de caso contrário: "mulher de meia-idade, após uma boa noite de sono, acorda no horário habitual e realiza todas as atividades de vida diária que costuma realizar rotineiramente e refere sentir-se disposta e com a energia habitual" (9).

e) Antecedentes e consequências da fadiga: os antecedentes da fadiga consistem em atividade física intensa, depressão, gestação e parto, presença de doenças e distúrbios do sono. Já as consequências compreendem letargia, sonolência; diminuição da motivação, atenção, paciência e concentração; sofrimento, necessidade extrema de descanso e mal-estar.

f) Referências empíricas: por ser um fenômeno subjetivo, os atributos só podem ser identificados através de menção pelo sujeito fatigado ou por comportamentos compatíveis com a evidência do fenômeno.

\section{MÉTODO DE ANÁLISE CONCEITUAL DE RODGERS}

O método de análise conceitual proposto por Rodgers ${ }^{(6)}$, conhecido como análise evolucionista, não difere, na essência, do elaborado por Walker e Avant ${ }^{(4)}$. Ambos iniciam com a seleção de um conceito de interesse; em seguida, identificação de usos e atributos do conceito, construção de casos-modelos e identificação de antecedentes e consequentes. A análise de conceito na perspectiva evolucionista faz-se necessária porque os conceitos são dinâmicos, "indistintos", dependentes do contexto e possuidores de alguma utilidade ou finalidade pragmática, devendo, portanto, ser continuamente refinados, de modo 
a serem introduzidas variações que venham favorecer sua clarificação e utilidade prática(6).

O método evolucionário de análise conceitual constitui um modo indutivo e descritivo de investigar o consenso relacionado ao conceito, o conhecimento histórico, assim como um instrumento para verificar área de concordância e discordância do uso do conceito entre as diversas disciplinas. Na visão evolucionária o conceito adquire significado através do seu uso e aplicação a cada situação no tempo, influenciado por fatores internos e externos que ampliam, refinam e caracterizam, adequadamente, esse conceito. A operacionalização da análise conceitual nessa perspectiva se dá mediante seis atividades que podem ocorrer concomitantemente, ou passo a passo ${ }^{6}$. A seguir, descreveremos essas atividades, considerando a análise do conceito condição crônica de saúde do adulto ${ }^{(10,11)}$.

a) Seleção do conceito de interesse: condição crônica de saúde, que foi selecionado dada a necessidade de promover sua compreensão dentro do contexto saúde.

b) Seleção do cenário apropriado para a coleta de dados e dos documentos a serem analisados. Considerando isso, foram escolhidas 41 fontes literárias, publicadas entre 1994 e 1998, abrangendo as áreas de Enfermagem, Medicina, Psicologia, Sociologia e Epidemiologia. c) Após a coleta e análise dos dados, foram identificados os atributos, os antecedentes, os consequentes, os conceitos relacionados (reúnem alguns dos atributos secundários que não coincidem com os essenciais) e a base contextual do conceito (variações interdisciplinares, socioculturais e temporais - ciclo evolutivo) - Figura 2.

d) A análise do ciclo evolutivo do conceito (1994 - 1998) permitiu a seguinte verificação: inicialmente, o conceito condição crônica de saúde foi nominado de doença crônica, sendo discutido, particularmente, no contexto das ciências biológicas, priorizando-se dados estatísticos, epidemiológicos e clínicos. Nas publicações mais recentes (1996, 1997, 1998), muitos autores, apesar de adotarem a abordagem biológica da condição crônica de saúde, ampliaram sua abordagem, incorporando, também, os pressupostos da epidemiologia. Nesse cenário, condição crônica é referida pelos autores como um evento no qual o indivíduo também pode "criar ou promover saúde", apesar da cronicidade. Do mesmo modo, a abordagem cultural (tradições, valores, saberes) e espiritual (possibilidade de enfrentamento) passaram a ser incluídas no conceito, em contraponto às verdades científicas da área biológica.

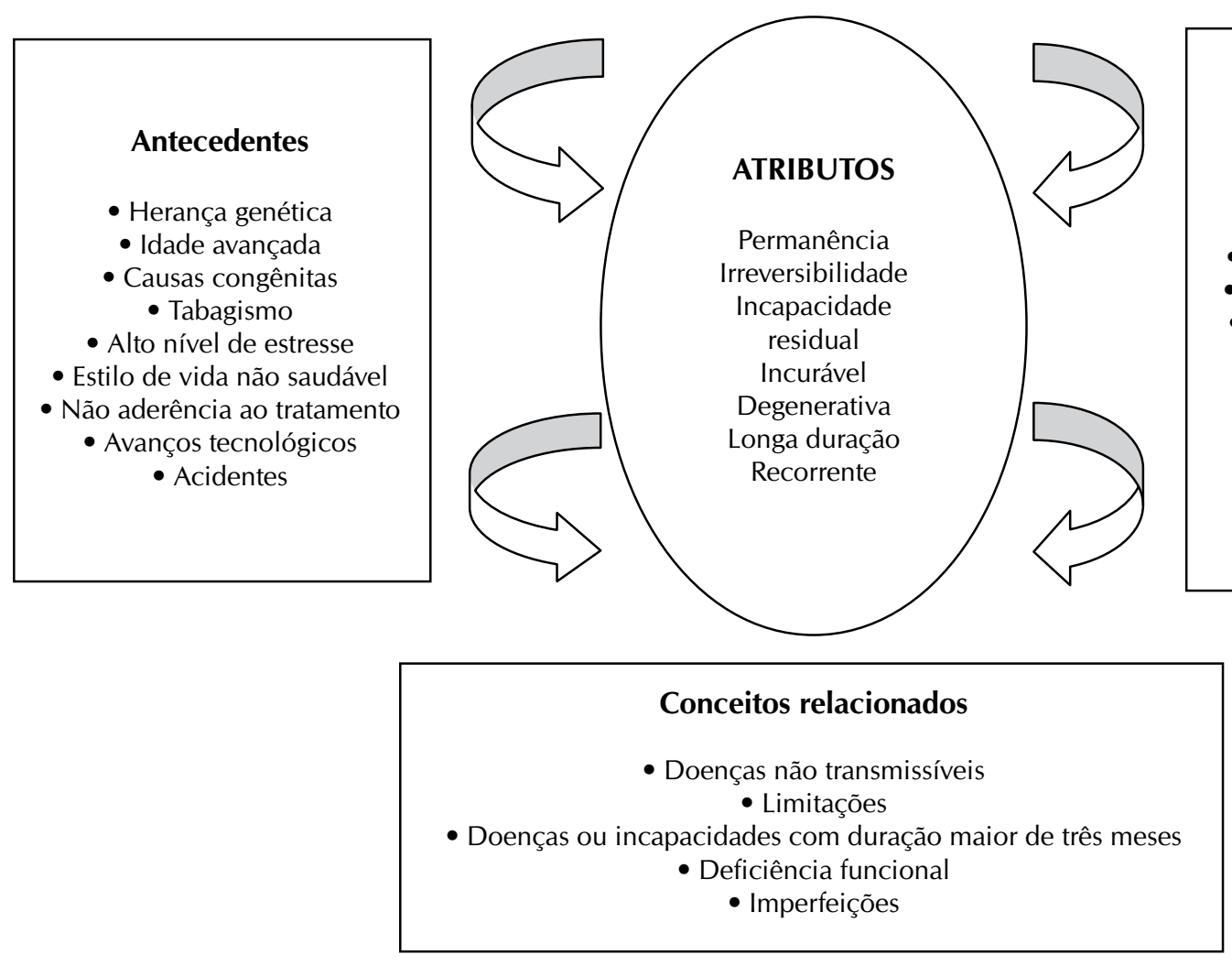

\section{Consequências}

- Modificações físicas, sociais e psicológicas

- Incapacidade e inabilidade

- Mudanças no estilo de vida

- Necessidade de adaptação e enfrentamento

- Desordens

músculo-esqueléticas, circulatórias

Figura 2 - Estrutura conceitual da condição crônica de saúde ${ }^{(11)}$. 
e) Caso-modelo: o relato a seguir traduz uma observação da "vida real" para demonstrar um caso exemplar do conceito condição crônica de saúde.

Maria, 45 anos, socialmente ativa e assídua ao trabalho, começou a observar que, mesmo em repouso, suas mãos apresentavam tremores associados à instabilidade postural. No início, apenas pensava, o que há de errado comigo? Mas, como o fato não comprometia suas atividades da vida diária, deixou para lá [...]. Nos meses subsequentes, os tremores se agravavam e outros sinais iam aparecendo com mais frequência, como: distúrbio de marcha e do sono, alteração na voz, desinteresse sexual, momentos de depressão e expressão facial em máscara. Frente a isso, Maria resolveu procurar um especialista e entender o que estava acontecendo com ela, quais as causas e o tratamento do problema, pois sempre fora muito saudável e desconhecia situações desse tipo em sua família. Acreditava que talvez fossem alterações decorrentes do envelhecimento precoce. Após avaliação, Maria recebeu esclarecimento de que, possivelmente, estava com "Doença de Parkinson", desordem degenerativa do sistema nervoso central [...], não sendo possível retardar sua progressão. Foi também orientada sobre a necessidade do uso contínuo de dopamina combinada - medicação que poderia causar distúrbios digestivos, psíquicos e cardiovasculares. Ela indagou sobre as perspectivas de cura com o tratamento e o modo que deveria agir, após iniciar a ingestão das drogas que, certamente, fariam desaparecer seus tremores. Após ter sua indagação respondida, Maria entristeceu-se, pois verificou que sua vida futura seria incerta. Indagava: "como farei para continuar trabalhando, uma vez que a doença ainda não tem cura e gera incapacidade?" Lamentava, também, o fato de que seu problema pudesse comprometer seus filhos. Comentou que iria rezar para que nada acontecesse a eles, pois não seria possível fazerem os testes antecipadamente, devido às condições financeiras da família ${ }^{(11: 8)}$.

\section{O MODELO DE ANÁLISE CONCEITUAL DE SWARTZ- BARCOTT E KIM}

O Modelo Híbrido de análise conceitual foi apresentado por Swartz-Barcott e $\mathrm{Kim}^{(7)}$. Este modelo interliga análise teórica e observação empírica, sendo composto de três fases que podem ser operacionalizadas de forma progressiva, fase a fase, ou de forma simultânea: fase teórica (seleção do conceito, busca e revisão da literatura, definição conceitual e operacional do conceito - instrumento de medida), fase de trabalho de campo (integração da análise teórica às observações empíricas referentes ao fenômeno no contexto em que se manifesta), que envolve o estabelecimento do cenário do estudo, a seleção de casos, a coleta e a análise de dados; e a fase analítica final, que compreende o cotejamento entre os dados resultantes da fase teórica inicial e as observações empíricas, sendo o produto final a apresentação da definição do conceito, bem como a identificação de lacunas conceituais que direcionam o desenvolvimento de outras pesquisas.
Considerando, de modo particular, esse cotejamento entre os dados teóricos e empíricos, Swartz-Barcott e Kim ${ }^{(7)}$ ressaltam que tal questionamento pode orientar o pesquisador: em que extensão a análise teórica e os achados empíricos apóiam a presença e a frequência do conceito na população investigada? ${ }^{(7)}$. Vários resultados podem ser obtidos a partir dessa análise: o significado atual do conceito pode ser apoiado ou meIhorado; uma definição diferente daquela previamente usada pode emergir; o conceito pode ser completamente definido, incluindo seu modo de mensuração. A seguir, apresentaremos uma análise do conceito Tensão do cuidador familiar de idosos dependentes, utilizando tal modelo ${ }^{(12)}$.

Este conceito foi selecionado para análise, dada a observação das pesquisadoras ${ }^{(12)}$, tanto na literatura como no âmbito do cuidado ao idoso dependente e cuidadores familiares, o uso de vários termos para sintetizar os efeitos evidenciados por esses cuidadores frente ao desempenho deste papel: sobrecarga, estresse, tensão - todos derivados da literatura internacional, em especial dos termos da língua inglesa: burden, stress, strain (caregiver burden, caregiver stress, caregiver strain). Assim sendo, a análise de tais conceitos na nossa cultura, proporcionaria aos profissionais de saúde e doutras áreas afins o suporte teórico-prático para sua aplicação na prática clínica junto ao idoso dependente e cuidador familiar.

$\mathrm{Na}$ análise do conceito em questão, a primeira fase, teórica, que tem como foco principal o desenvolvimento de uma fundamentação científica para subsidiar a análise e o refinamento do conceito nas fases subsequentes, foi efetivada através de um corpus de 84 trabalhos, produzidos por diferentes disciplinas da área de saúde, publicados nos idiomas Português e Inglês no período de 1990 a 2000. Na análise dessa literatura foi verificado que os termos sobrecarga do cuidador, estresse do cuidador e tensão do cuidador evidenciavam diferenças conceptuais e etimológicas sutis, o que justificavam seu uso como sinônimos e acarretavam dificuldades para a compreensão adequada dos efeitos negativos da provisão de cuidados, porém, o mais aplicável a nossa cultura compreendia o termo tensão do cuidador, passando este a ser o foco da investigação ${ }^{(12)}$. Cabe destacar que os resultados dessa análise subsidiaram, ainda, a elaboração do instrumento de coleta de dados empíricos (que comportou categorias de dados relativos aos antecedentes, atributos e consequências do conceito Tensão do cuidador familiar de idosos dependentes) e, consequentemente, a fase de trabalho de campo.

A segunda fase, de campo, envolveu uma amostra intencional de trinta cuidadoras de familiares idosos dependentes que, durante Consulta de Enfermagem ao binômio cuidadora/ idoso, evidenciavam efeitos deletérios atribuídos ao desempenho do papel de cuidadora do parente idoso. Após esse primeiro momento, os demais contatos com as cuidadoras foram efetivados em seus domicílios que, na maioria dos casos, também abrigavam o idoso dependente, constituindo, portanto, o ambiente natural do cuidado.

A análise conceitual guiada pelo Modelo Híbrido requer, prioritariamente, uma atitude reflexiva e interpretativa, demandando do analista uma maior aproximação com as interações sociais e ambientais dos participantes do estudo, 
refletindo, revisando significados e verificando o que ocorre. A coleta de dados se deu mediante entrevista individual, subsidiada pelo instrumento ora referido, realizada em dia e horário agendados previamente, considerando a disponibilidade das participantes do estudo ${ }^{(12)}$.

Terminada a fase de campo, foi iniciada a fase analítica final, com realização de uma interface entre os dados resultantes da fase teórica inicial e os dados e observações empíricos. Esse procedimento permitiu confirmar a definição conceitual elaborada para o fenômeno Tensão do cuidador familiar de idosos dependentes, como sendo "um estado dinâmico de alteração do bem-estar do cuidador familiar, variável de pessoa para pessoa, multideterminado e cumulativo, resultante do processo de cuidado do idoso dependente" (12:472).

Quanto aos fatores antecedentes que, atuando de modo inter-relacionado, podem determinar diferentes níveis de tensão no cuidador, foram observados aqueles relacionados às características sócio-demográficas do cuidador (sexo, idade, renda e nível educacional); aos déficits do idoso (físicos, cognitivos e comportamentais); a interação idoso/cuidador (relação próxima de parentesco, problemas de relacionamento); ao ambiente (disfunção familiar pregressa, suporte social reduzido ou ausente, ambiente físico inadequado para o cuidado, materiais e equipamentos insuficientes para o cuidado) e às demandas de cuidado (provisão contínua de cuidado, cuidado direto ou instrumental e sensação de sobrecarga) ${ }^{(13)}$.

No que concerne aos atributos, verificou-se que a Tensão do cuidador familiar de idosos dependentes constitui um fenômeno multidimensional que se evidencia por alterações biopsicossociais, tendo como atributos críticos (presentes em $80 \%$ a $100 \%$ dos casos) alterações no estado físico - doenças/sintomas psicossomáticos e/ou agravamento de doenças prévias; alterações emocionais - depressão, ansiedade, baixa autoestima situacional, pesar e irritabilidade; desequilíbrio entre atividade/repouso - fadiga e déficit de lazer; além de enfrentamento individual comprometido - baixo controle sobre a situação de cuidado e pouca adesão ao autocuidado ${ }^{(12)}$.

No referente às consequências, foram identificados: alterações na dinâmica familiar (conflitos familiares, maus-tratos ao idoso, problemas nas relações maritais, mudanças nas rotinas e papéis da família) e interferência na vida social da cuidadora (isolamento social, alterações nos projetos pessoais/profissionais, dificuldades financeiras). As consequências podem, também, indiretamente, causar tensão, por afetar de maneira adversa as condições de vida, experiências vividas e necessidades sentidas pela cuidadora e, por sua vez, realimentar a continuidade do processo.

A análise conceitual subsidiada pelo Modelo Híbrido de Swartz-Barcott e $\mathrm{Kim}^{(7)}$ não inclui a apresentação de caso que expresse um modelo do conceito. No entanto, suas autoras valorizam a criatividade ou inovação do pesquisador, desde que o resultado final deste procedimento seja a máxima compreensão do conceito. Assim, um caso modelo ou exemplar, se desenvolvido, deve ser efetivado a partir das observações teóricas e empíricas referentes ao fenômeno, e não "inventado"(7).

\section{CONSIDERAÇÕES FINAIS}

Ante a discussão ora apresentada, ressaltamos a necessidade de novas reflexões e atualizações no concernente as metodologias de análise conceitual, favorecendo, dessa forma, a clarificação de conceitos úteis para a prática de enfermagem, pois a organização do conhecimento sobre tais conceitos poderá contribuir para sua adequada aplicabilidade nos diferentes cenários de cuidado. Além disso, a análise conceitual está intimamente relacionada à evolução e expansão de conhecimentos na Enfermagem.

Considerando que os conceitos advêm da prática cotidiana, faz-se necessário lembrar que é por meio das observações apreendidas e refletidas na dinâmica desse cotidiano vivido no âmbito da nossa prática de enfermagem, bem como ante as modificações contínuas e complexas do conhecimento científico, que emerge a necessidade da análise contínua dos conceitos de nosso interesse pois, através de tal procedimento, podemos experimentar intercâmbio entre nossos próprios saberes e os saberes dos diferentes campos disciplinares, tornando-se possível explicar a natureza da Enfermagem, definir seu campo específico e sua metodologia científica.

\section{REFERÊNCIAS}

1. Poles K, Bousso RS. Morte digna da criança: análise de conceito. Rev Esc Enferm USP 2009; 43(1): 215-22.

2. Mcewen M, Wills EM. Bases teóricas para a enfermagem: os fundamentos à prática profissional. $2^{\mathrm{a}}$ ed. Porto Alegre (RS): Artmed; 2009

3. Morse JM. Exploring the theoretical basis of nursing using advanced techniques of concept analysis. Adv Nurs Sci. 1995; 17 (3): 31-46.

4. Walker LO, Avant KC. Concept development. In: Walker LO, Avant KC. Strategies for theory construction in nursing. 3rd ed. Norwalk: Appleton \& Lange; 1995.
5. Burnell L. Compassionate care: a concept analysis. Home health care manag. pract. 2009; 21(5): 319-24.

6. Rodgers LB. Concept analysis: an evolutionary view. In: Rodgers BL, Knalf AK. Concept development in nursing: foundations, techniques, and applications. 2nd ed. Philadephia: Saunders; 2000.

7. Schwartz-Barcott D, Kim HS. An expansion and elaboration of the hybrid model of concept development. In: Rodgers BL, Knalf KA. Concept development in nursing: foundations, techiniques, and applications. 2nd ed. Philadelphia: Saunders; 2000. 
8. Wilson J. Thinking with concepts. New York (NY): Cambridge University Press; 1963.

9. Mota DDCF, Cruz DALM, Pimenta CAM. Fadiga: uma análise do conceito. Acta Paul Enferm 2005; 18(3): 285-93.

10. Freitas MC, Mendes MMR. Condição crônica: análise do conceito no contexto da saúde do adulto. Rev. Latino-am Enfermagem 2007; 15(4): 590-7.

11. Freitas MC. Condição crônica de saúde do adulto: análise do conceito. [Dissertação de Mestrado]. São Paulo (SP):
Escola de Enfermagem de Ribeirão Preto/USP; 1999.

12. Fernandes MGM, Garcia TR. Estrutura conceitual da tensão do cuidador familiar de idosos dependentes. Rev. Eletr. Enf. [periódico on line]. 2009; 11(3): 469-76. Disponível em: http://www.fen.ufg.br/revista/v11/n3v$11 \mathrm{n} 3 \mathrm{a} 02 . \mathrm{htm}$.

13. Fernandes MGM, Garcia TR. Determinantes da tensão do cuidador familiar de idosos dependentes. Rev Bras Enferm, 2009; 62(1): 57-63. 